\title{
Leibniz rule and exact supersymmetry on lattice: a case of supersymmetrical quantum mechanics
}

\author{
Mitsuhiro Kato \\ Institute of Physics, University of Tokyo, Tokyo 153-8902, Japan \\ E-mail: kato@hep1.c.u-tokyo.ac.jp
}

\section{Makoto Sakamoto}

Department of Physics, Kobe University, Rokkodai, Nada, Kobe 657-8501, Japan

E-mail: dragon@kobe-u.ac.jp

\section{Hiroto So*}

Department of Physics, Niigata University, Ikarashi 2-8050, Niigata, 950-2181, Japan

E-mail: so@muse.sc.niigata-u.ac.jp

\begin{abstract}
We propose a new formulation of lattice theory. It is given by a matrix form and suitable for satisfying Leibniz rule on lattice. The theory may be interpreted as a multi-flavor system. By realizing the difference operator as a commutator, we may obtain exact supersymmetric theories on lattice explicitly. Some problems such as locality and single flavor reduction are also commented.
\end{abstract}

XXIIIrd International Symposium on Lattice Field Theory

25-30 July 2005

Trinity College, Dublin, Ireland

\footnotetext{
${ }^{*}$ Speaker.

$\dagger$ This work is supported in part by the Grants-in-Aid for Scientific Research No. 17043004-0167 from the Japan Society for the Promotion of Science.
} 


\section{Motivations}

Supersymmetry(SUSY) is necessary for understanding unified field theories in particle physics. The nonperturbative effects such as the vacuum property and SUSY breaking terms of unified theories take us back to our real world . To investigate the effects, we must realize full (or a part of) SUSY algebra in constructing exact SUSY on lattice. In the free theories, it is realized by a relation between fermion and boson propagators. On the other hand, a problem of Leibniz rule must be solved in the interacting theories[1].

Our solution for the problem is a theory of matrix-valued fields and the realization of a difference operator as a commutator. A whole lattice is treated as a system just like a 'field'. After a decomposition of the matrix into a lattice field, it may be seen as a multi-flavor system.

Kaplan et al. have approached by imposing orbifolding conditions on a matrix [2, 3, 4, 5]. But they did not define the difference operator in a meaning of matrix. In the context of noncommutative geometry, Bars and Minic presented a matrix-type theory[6]. They constructed a 2-dimensional theory on a matrix to obtain the non-commutative property.

In this talk, we set a 1-dimensional lattice theory on a matrix, which covers more general construction ways. A Wess-Zumino model in a 1-dimensional case is constructed for simplicity. This is extendable to 2- and 4-dimensional cases.

\section{Our Formalism}

Our formulation is an extension of the ordinary lattice theory and to treat lattice fields as a whole. We consider a matrix-valued field $\phi_{i j}$, where $i, j=1, \cdots N$ and $N$ is a 1-dimensional lattice size. The product of matrix-valued fields follows to a usual matrix product rule. The diagonal component, $\phi_{i, i}$ is recognized as a field on a site, $i$. Furthermore, a field on a link is realized as $\phi_{i, i+1}$. The trace operation corresponds to the space-time summation.

The important key of our formulation is the definition of a difference operator. A commutator with a constant matrix, $d$ is identified as the operator,

$$
i[d, \phi] \rightarrow \nabla \phi
$$

where $\nabla$ is a certain difference operator.

For the choice of $d$, the following conditions are imposed;

(1) hermitian,

(2) local,

(3) simple.

The explicit candidate for $d$ is

$$
d=\frac{1}{2 i}\left(T^{+}-T^{-}\right),
$$

where $T^{+}$is a forward displacement operator and $T^{-}$is a backward displacement operator; 


$$
T^{+}=\left(\begin{array}{cccccc}
0 & 1 & 0 & \cdots & \cdots & 0 \\
\vdots & \ddots & 1 & \ddots & \ddots & \vdots \\
\vdots & \ddots & \ddots & \ddots & \ddots & \vdots \\
\vdots & \ddots & \ddots & \ddots & 1 & 0 \\
0 & \ddots & \ddots & \ddots & \ddots & 1 \\
1 & 0 & \cdots & \cdots & \cdots & 0
\end{array}\right),
$$$$
T^{-}=\left(\begin{array}{cccccc}
0 & \cdots & \cdots & \cdots & 0 & 1 \\
1 & \ddots & \ddots & \ddots & \ddots & 0 \\
0 & 1 & \ddots & \ddots & \ddots & \vdots \\
\vdots & \ddots & \ddots & \ddots & \ddots & \vdots \\
\vdots & \ddots & \ddots & 1 & \ddots & \vdots \\
0 & \ldots & \ldots & 0 & 1 & 0
\end{array}\right) .
$$

Instead of taking the matrix form, our formulation may be interpreted as a multi-flavor system, which is useful in understanding Leibniz rule on lattice.

A $(2 N-1)$-flavored field is defined as

$$
\phi_{k}(n)=\phi_{i, j},
$$

where a flavor index, $k \equiv j-i$ and a lattice site, $n \equiv i+j$. From Eq.(2.1) and Eq.(2.2), the difference operator for a flavored field becomes to

$$
\frac{1}{2}\left(\nabla^{+}+\nabla^{-}\right)\left(\phi_{k-1}+\phi_{k+1}\right)
$$

where $\nabla^{+}$means a forward difference and $\nabla^{-}$does a backward difference. It should be noted that the flavor indices are shifted. A matrix-valued theory and a multi-flavored system are summarized in Table 1.

Table 1. Correspondence among various theories.

\begin{tabular}{|c||c|c|c|}
\hline \multicolumn{1}{|c||}{ continuum theory } & lattice theory & matrix - valued theory & multi - flavored theory \\
\hline \hline a field & $\phi_{n}$ & $\phi_{i, j}$ & $\phi_{k}(n)$ \\
\hline coordinate & $n$ & $i$ & $n=i+j$ \\
\hline flavor & single flavor & single flavor & $(2 N-1)-$ flavored system \\
\hline space integral & $\sum_{n}$ & $\operatorname{tr}$ & $\sum_{n}$ \\
\hline a scalar field & $\phi_{n}$ & $\phi_{i, i}$ & $\phi_{0}(n)$ \\
\hline$\times$ & link fields $\phi_{n, \pm \hat{1}}$ & $\phi_{i, i \pm 1}$ & $\phi_{ \pm 1}(n)$ \\
\hline$\times$ & fields on double - length link & $\phi_{i, i \pm 2}$ & $\phi_{ \pm 2}(n)$ \\
\hline derivative & difference & $i[d, \phi]$ & $\frac{1}{2}\left(\nabla^{+}+\nabla^{-}\right)\left(\phi_{k-1}+\phi_{k+1}\right)$ \\
\hline
\end{tabular}

\section{Leibniz Rule}

In this section, we clear the problem of Leibniz rule and show how a commutator formulation or multi-flavor interpretation may solve the problem. A differential operator has generally the following properties: (1) linearity, (2) Leibniz rule, and (3) normalization or conjugation property for an independent variable. From the properties, we may differentiate any analytic function.

The second condition on lattice causes a problem. For example,

$$
\nabla^{+}\left(\phi_{n} \psi_{n}\right)=\left(\nabla^{+} \phi_{n}\right) \psi_{n+1}+\phi_{n}\left(\nabla^{+} \psi_{n}\right) \neq\left(\nabla^{+} \phi_{n}\right) \psi_{n}+\phi_{n}\left(\nabla^{+} \psi_{n}\right)
$$


The first identity is not suitable for constructing exact SYSY theories. This situation is caused under more general situation. The local lattice theory may not hold exact Leibniz rule. On the other hand, a commutation relation between matrices is always distributive. In our formalism, simple Leibniz rule holds,

$$
[d, \phi \psi]=[d, \phi] \psi+\phi[d, \psi] .
$$

In the multi-flavored system, the shift of the flavor is essential. Some comments are put in order; for single flavor case, Leibniz rule and locality are incompatible in lattice theory[1], our Leibniz rule for product of functions is similar to that of non-commutative differential geometry[6] .

\section{Interacting SUSY Theory}

In the section, we construct an interacting SUSY model on lattice and represent exact SUSY. Although our explicit example is the model in 1 dimension, higher dimensional cases are mentioned in the summary. We prepare a scalar matrix, $\phi$, fermi matrices, $\bar{\psi}, \psi$, and an auxiliary matrix, $F$.

Our total action, $S$ is decomposed into the three parts on off-shell formalism;

$$
S=\operatorname{tr} L=S_{0}+S_{1}+S_{\text {int }},
$$

where the free part is written as

$$
S_{0}=\operatorname{tr}\left(-\frac{1}{2}[d, \phi]^{2}-\frac{i}{2}(\bar{\psi}[d, \psi]-[d, \bar{\psi}] \psi)-\frac{1}{2} F^{2}\right),
$$

a doubling-avoiding (Wilson-like) action is

$$
S_{1}=\alpha \operatorname{tr}(F\{M, \phi\}+\{\bar{\psi}, M\} \psi),
$$

and the interacting terms $S_{\text {int }}$ are

$$
S_{\mathrm{int}}=\operatorname{tr}\left(\frac{\lambda}{2 !} F \phi^{2}+\frac{\lambda}{2 !} \sum_{s=0}^{1} \bar{\psi} \phi^{s} \psi \phi^{1-s}\right) .
$$

It is noted that $\alpha$ is a nonzero constant and $M \equiv 2-T^{+}-T^{-}$.

The action is invariant under the following SUSY transformation with Grassman-odd parameters, $\varepsilon$ and $\bar{\varepsilon}$;

$$
\begin{gathered}
\delta \phi=-(\bar{\psi} \varepsilon+\bar{\varepsilon} \psi), \\
\delta \psi=\varepsilon(i[d, \phi]+F), \\
\delta \bar{\psi}=\bar{\varepsilon}(-i[d, \phi]+F)
\end{gathered}
$$

and

$$
\delta F=\varepsilon(-i[d, \bar{\psi}])+\bar{\varepsilon}(-i[d, \psi]) .
$$

This transformation satisfies an SUSY algebra;

$$
\left[\delta_{\bar{\varepsilon}}, \delta_{\varepsilon}\right] \mathscr{O}=2 i \bar{\varepsilon} \varepsilon[d, \mathscr{O}]
$$


where $\mathscr{O}$ is any field such as $\phi, \psi, \bar{\psi}$ and $F$.

The variation of the lagrangian, $L$ may be written as

$$
\delta L=[d, \mathscr{O}],
$$

where

$$
\begin{gathered}
\mathscr{O}=\frac{1}{2} \bar{\psi} \varepsilon[d, \phi]+\frac{1}{2} \bar{\varepsilon}[d, \phi] \psi+\frac{i}{2}(\bar{\varepsilon} F \psi)-\frac{i}{2}(\bar{\psi} \varepsilon F) \\
-i \alpha \bar{\varepsilon} \psi\{M, \phi\}-i \alpha \varepsilon \bar{\psi}\{M, \phi\}-i \frac{\lambda}{2 !} \varepsilon\{\bar{\psi}, \phi\} \phi-i \frac{\lambda}{2 !} \bar{\varepsilon}\{\psi, \phi\} \phi .
\end{gathered}
$$

Owing to this ristricted writing space, we have written these terms shorthandly. The actual expression in Eqs. (4.1) and (4.7) must be careful in the matrix-ordering. From Eq. (4.6), it is clear that our action is invariant under the transformation,

$$
\delta S=\delta \operatorname{tr} L=\operatorname{tr}[d, \mathscr{O}]=0
$$

\section{Summary}

In this talk, we have presented a new formulation of lattice theory. This formulation is to consider a matrix as whole lattice field. Our proposal of matrix-valued field theory is different from that of Kaplan et al. [2, 3, 4, 5] in points of the difference operator and the correspondence to the ordinary lattice fields. The operator in our case is realized as a commutator with a constant matrix. The Leibniz rule always holds in this formulation because the commutator has a distributative property. An explicit model with the exact SUSY has been constructed in 1 dimension. It is possible to extend to higher dimensional theories [7] such as

$$
\phi_{i, j} \rightarrow \phi_{\left(i_{1}, i_{2}\right),\left(j_{1}, j_{2}\right)}
$$

in 2 dimensions. Non chiral theories are realized in $D=2,4$ theories owing to avoiding doubling or Wilson-like terms [7].

The important problem is the continuum limit of our theory. Although the limit operation is a difficult problem, we must comment on a flavor-reduction and locality. Our theory has a (2N-1)flavored Wess-Zumino model in 1 dimension on lattice. The reduction to a single flavored model must be done with keeping the exact SUSY invariance. The consistency between some constraints for matrices and the invariance or the commutator with $d$ is an essential point. Nonlocal terms are generally induced from the reduction. Nevertheless, there are some circumstantial evidence for us to control its locality. The first evidence is that the free part of our action may be reduced to a single flavored system with only local terms. The second is the transformation (4.2), (4.3), (4.4), (4.5) which is written by only local terms. The final one is the existence of a local Nicolai map in 1- and 2-dimensional models. The dynamical properties such as phase structure may find the expected reduction.

In the remaining problems, inclusion of gauge interactions for our theory and construction of chiral theories are nontrivial matter specially. 


\section{References}

[1] P.H. Dondi and H. Nicolai, Lattice Supersymmetry, Nuovo Cimento A4 (1977) 1.

[2] D.B. Kaplan, E. Katz and Mithat Unsal, Supersymmetry on a Spatial Lattice., JHEP 05 (2003) 037 [hep-lat/0206019].

[3] A.G. Cohen, D.B. Kaplan, E. Katz and M. Unsal, Supersymmetry on a Euclidean Space-Time Lattice. 1. a Target Theory with Four Supercharges, JHEP 08 (2003) 024 [hep-lat / 0302017 ].

[4] A.G. Cohen, D.B. Kaplan, E. Katz and M. Unsal, Supersymmetry on a Euclidean Space-Time Lattice. 2. Target Theories with Eight Supercharges, JHEP 12 (2003) 031 [hep-lat/0307012].

[5] D.B. Kaplan, and M. Unsal, a Euclidean Lattice Construction of Supersymmetric Yang-Mills Theories with Sixteen Supercharges, [hep-lat/0503039].

[6] I. Bars and D.Minic, Noncommutative Geometry on a Discrete Periodic Lattice and Gauge Theory, Phys. Rev. D62 (2000) 105018, [hep-th/9910091].

[7] M.Kato, M. Sakamoto and H. So, in preparation. 